\title{
Challenges in Teaching Praxis When CAS Is Used in Upper Secondary Mathematics
}

\author{
Niels Groenbaek, Claus Larsen, Henrik Bang, Hans-Georg Weigand, \\ Zsolt Lavicza, John Monaghan, M. Kathleen Heid, Mike Thomas \\ and Paul Drijvers
}

The DG focused on the relationship between CAS and mathematics in teaching and learning, educational design and the qualification of teachers' choices concerning the use and non-use of CAS.

\section{Headword Summary}

The first DG session was on challenges and opportunities for design, with main themes:

- Reasons for CAS not being more widespread. Reports as far back as the first ICMI studies in the mid 80s were quite optimistic, and although there have been many investigations and modes of development, the situation today is that CAS-use is supported by the same basic ideas. But optimism has faded, mainly because the technical difficulties with CAS are underestimated and CAS is not just an isolated tool but represents an entire environment.

- Documentation and assessment with CAS has proved to be difficult.

- Gainful CAS-use requires an overarching concept of teaching and learning as CAS changes mathematics in several directions - content, style and activities, exemplified by: Content-CAS's enlarged look-up access to knowledge has changed the focus on what is important to teach and what is not; style-CAS shifts the view on what to prove and how; student activities - a shift from more or less demanding problem solving techniques to the logic of solution methods and checking modes.

- Teachers' knowledge about aspects of the underlying mathematics that CAS may reveal, e.g. to what extent, if any, should one master complex numbers and

N. Groenbaek $(\bowtie)$

University of Copenhagen, Copenhagen, Denmark

e-mail: gronbaek@math.ku.dk 
deal with or dismiss them didactically, because students encounter them solving quadratic equations. Similarly, the probability that students produce unexpected results increases with CAS, so the teacher decision between giving immediate response or suspending answers allowing for more reflection on the issue in an increased challenge.

The second DG day focused on in-service training, didactical discourse and changes in teacher role:

- The big diversity in teachers' implementation of CAS on one hand makes it difficult to generate common standards but, on the other hand, offers a range of experiences to draw on. Instigation of teacher reflection on CAS and mathematics and the choices made. Coaching provides one option; another is the development of communities where you can share experiences.

- CAS changes and creates situations in the classroom. Teachers are faced with a much wider range of on-spot choices and solutions. As a consequence, the importance of the teacher's exemplary work has increased.

- Examples of how a comprehensive pool of teaching ideas with CAS are available for teachers, combined systematically with teachers' training.

- Examples of how to develop tools used for electronic marking of CAS assignments, taking differing correct answer options, differing notation etc. into account.

Open Access Except where otherwise noted, this chapter is licensed under a Creative Commons Attribution 4.0 International License. To view a copy of this license, visit http://creativecommons. org/licenses/by/4.0/.

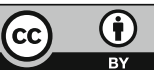

